\title{
Isolation of mumps virus from the inner ear after sudden deafness
}

\author{
G A WESTMORE, B H PICKARD, H STERN
}

British Medical fournal, 1979, 1, 14-15

\section{Summary and conclusions}

A 26-year-old woman with bilateral otosclerosis underwent right stapedectomy with an excellent result. One year later, however, she developed symptoms of mumps and within two days was completely deaf in the right ear. Prompt surgical exploration excluded a complication of the otosclerosis and a perilymph fistula, but culture of a sample of perilymph grew mumps virus.

The case provides direct evidence of a relation between mumps virus infection and inner-ear damage.

\section{Introduction}

The association of mumps virus infection with the rapid onset of severe or total sensorineural deafness has been appreciated for over 100 years. ${ }^{1}$ Perceptive deafness due to mumps is more common than generally recognised. It is usually partial and unilateral and is generally followed by recovery, but total irreversible deafness also occurs: among 298 servicemen in Finland with mumps parotitis there were 13 cases of deafness $(4 \%)$, one of which progressed to complete and constant loss of hearing in one ear. ${ }^{2}$ Mumps is a common infection and, as in meningoencephalitis, deafness may be the sole clinical manifestation, being unaccompanied by an obvious swelling of salivary glands. ${ }^{3}$ It is therefore surprising to find only scanty mention of this complication outside otology; most reference works of pathology describe only orchitis, meningoencephalitis, and pancreatitis, which although more dramatic may ultimately be less devastating.

Hitherto deafness due to mumps has been diagnosed either clinically, from preceding enlargement of salivary glands, or by detecting a rising titre of mumps antibodies. ${ }^{3}$ Histopathological findings in the ear are generally non-specific and do not distinguish mumps damage from that caused by other viruses, such as measles or rubella. ${ }^{4} \mathrm{By}$ an unusual combination of events we were able to obtain perilymph from the inner ear of a patient for virological studies within a few days of the onset of deafness, providing what we believe to be the first direct evidence for the aetiological relation between mumps virus infection and innerear damage.

\section{Case report}

A 26-year-old housewife presented in February 1976 with a history of gradual onset of bilateral deafness over three to four years. She also complained of bilateral tinnitus but there were no other symptoms and no relevant occupational or environmental factors. Her tympanic membranes were normal and there was conduction deafness of 40-50 db. Bilateral otosclerosis was diagnosed. A hearing aid was prescribed

St George's Hospital and Medical School, London SW1

G A WESTMORE, MB, FRCS, senior registrar in otolaryngology B H PICKARD, MB, FRCS, consultant in otolaryngology H STERN, MB, FRCPATH, professor of virology

for the left ear and she was admitted in June 1977 for right stapedectomy. At operation the diagnosis was confirmed, the posterior twothirds of the stapes footplate removed, and a $4.5 \mathrm{~mm}$ standard Teflon piston inserted between the long process of the incus and the perforated oval window. A plug of autogenous fat was placed in the oval window around the shaft of the piston to prevent leakage of perilymph. Postoperative recovery was uneventful, with excellent restoration of hearing (fig 1).

$\underline{a}$

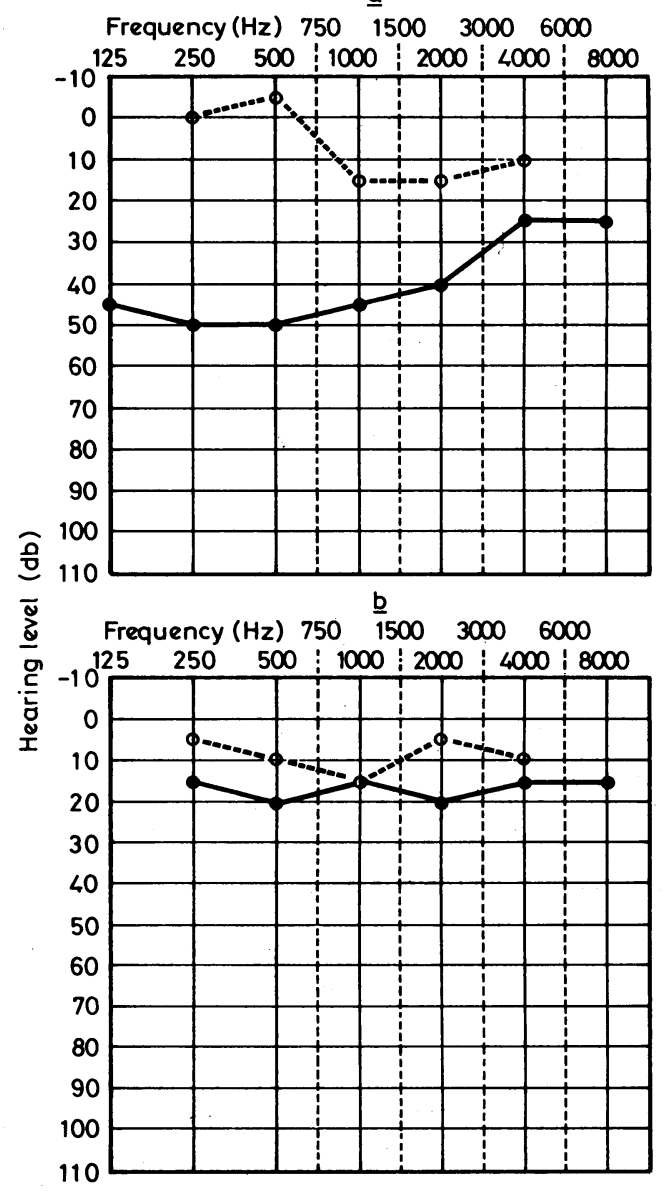

FIG 1 -Hearing level in right ear before $(a)$ and after $(b)$ stapedectomy. - Air conduction. $0 . .-0$ Bone conduction.

There were no problems until June 1978, when the patient became unwell, with general malaise, sore throat, and painful bilateral parotid swellings. She was not aware of having been in contact with mumps, but mumps was apparently prevalent in her neighbourhood. Two days later she noticed right-sided hyperacusis and, later the same day, deterioration of hearing in the right ear, which progressed to total loss over about four hours. Tinnitus persisted in the right ear, and next day she developed moderate vertigo associated with nausea and aggravated by postural change.

She was seen at this hospital three days after the onset of deafness and was found to have total right-sided sensorineural hearing loss; hearing in the left ear showed no appreciable change from that recorded a year before (fig 2). The tinnitus and nausea had gone. She 


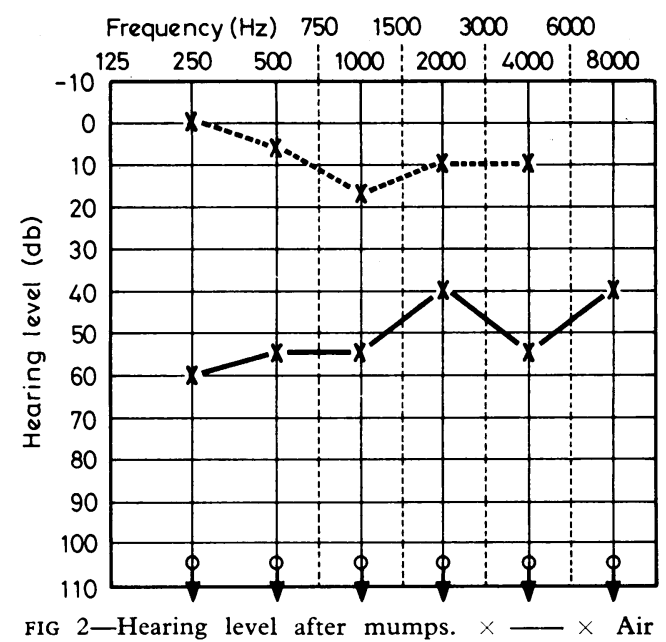

conduction in left ear. left ear. $\rho$ No response in right ear.

was admitted to hospital immediately for exploration of her right ear because, although the symptoms were suggestive of cochlear damage due to mumps, sudden deafness may result from cochlear otosclerosis or from a perilymph fistula occurring after stapedectomy, which can sometimes be corrected by prompt action. There was no evidence of fistula formation, however, and, as what could be seen of the membranous labyrinth under the high power of the operating microscope appeared to be normal, the Teflon piston was removed and a sample of perilymph (about $0.1 \mathrm{ml}$ ) aspirated for virological examination. The vertigo resolved after four weeks but hearing did not return.

Four days after the operation the patient's sister, who had been in contact with her about 14 days before, developed mumps, diagnosed by her general practitioner.

Virology-Culture of the perilymph in primary monkey kidney tissue cultures grew mumps virus; a haemadsorbing agent was recognised after 10 days' culture and identified as mumps virus by haemadsorption-inhibition with specific hyperimmune animal serum. A sample of the patient's serum collected eight weeks after the operation had a mumps $\mathrm{V}$ complement-fixing antibody titre of 64 and an $\mathrm{S}$ antibody titre of 16 .

\section{Comment}

Sudden deafness apparently related to concurrent mumps infection is not rare in otological practice but we have not previously had justification for exploring the affected ear. This patient, however, had been operated on for otosclerosis and, despite her mumps, the possibility could not be ignored that her sudden loss of hearing was actually a complication of the otosclerosis or of the corrective operation and might be remedied by emergency surgery. There was also the question of preventing meningitis, which could result from a perilymph fistula occurring after stapedectomy. Neither audiometry nor electrocochleography, which identify accurately the site of a sensorineural hearing loss, would have helped in diagnosis; perilymph fistula and cochlear otosclerosis both produce cochlear deafness, and viruses cause either cochlear or retrocochlear hearing loss or both by damaging the hair cells of the organ of Corti or the myelin sheath of the eighth cranial nerve or both. At the same time, since recovery from total deafness caused by mumps is rare $^{5}$ we thought that the chances of compromising the ear by performing an unhelpful operation were far outweighed by the other arguments.

The operation afforded the exceptional opportunity to obtain a sample of perilymph only five days after the onset of illness, and the isolation of mumps virus directly from the inner ear substantiates Toynbee's original observation ${ }^{1}$ that mumps is a cause of deafness. Whether the stapedectomy operation predisposed to further damage of the ear by mumps virus remains uncertain.

\section{References}

1 Toynbee, J, The Diseases of the Ear. London, John Churchill, 1860.

2 Vuori, M, Lahikainen, E A, and Peltonen, T, Acta Oto-laryngologica, 1962, 55, 231.

${ }^{3}$ Rowson, K E K, and Hinchcliffe, R, British fournal of Audiology, 1976, 10, 107.

${ }^{4}$ Lindsay, J R, Acta Oto-laryngologica, 1967, 63, 138.

5 Scott-Brown, W G, in Diseases of the Ear, Nose and Throat, ed J Ballantyne and J Groves, vol 2, p 439. London, The Butterworth Group, 1971.

(Accepted 25 October 1978)
ONE HUNDRED YEARS AGO During the last month, smallpox has continued to be increasingly prevalent in the metropolis. In the four weeks ended on Saturday last, 52 deaths from that disease were registered; and 261 fresh cases admitted to the Metropolitan Asylums Hospitals, which contained on the 11th instant as many as 267 patients. In the middle of October, when the epidemic had reached its lowest point, the hospitals contained only a third of this number of patients; but the totals have since gone on increasing every week, and during the four weeks under consideration were 194, 225, 254 , and 267 respectively. How much of this prevalence owes its origin to the inadequate arrangements for the enforcement of vaccination in the metropolis, needs hardly be stated. The Local Government Board, indeed, in the letter which they addressed to the Metropolitan Asylums Managers on the 23rd August last, ascribed a greater part of the blame on this cause, and said that much more would be necessary to put the arrangements of the metropolis on a satisfactory footing. They added that they were not relaxing their efforts to complete this; but we confess we should like to see some outward and visible signs of the energy which the Board have assumed for themselves. When nearly four thousand deaths occur within two years in the metropolis at a time when vaccination is supposed to be more rigidly looked after, and more extensively and carefully practised, than it ever was before, it must be admitted that there is a hitch somewhere; and we shall be much surprised if experience do not show that the Local Government Board made an egregious mistake in evading the inquiry pressed upon them by the Asylums Managers in the interests of the community at large. It is scarcely necessary to observe that the evils of the present unsatisfactory vaccination arrangements of the metropolis are not confined to London itself; cases of small-pox are exported from town into various country districts, and we have come across innumerable references in reports of medical officers of health to minor epidemics started in this way. The constant communication which London has with all parts of the United Kingdom, indeed with the whole world, renders it of vital importance that its power of dissemination of a disease so infectious as small-pox should be brought to the lowest possible point. If there be, and doubtless there are, exceptional difficulties in the circumstances of London as regards small-pox, so ought exceptional measures to be taken to insure the proper carrying out of the Vaccination Acts therein; and, until the Local Government Board rise to the situation, we cannot hope for any appreciable diminution in the present mortality from small-pox in the metropolis.-It may be convenient to give in this place the statistics contained in the last two returns of the Registrar-General with regard to the prevalence of small-pox in London. In 1877 and 1878, the fatal cases registered were 3960 , of which 2544 were recorded in 1877 , and 1416 in 1878. The annual death-rate from small-pox in the two years was thus equal to 55 per 100000 of the estimated population. After distributing the fatal hospital cases, the death-rate from small-pox was equal to 25 per 100000 in the central, 37 in the west, 53 in the north, 60 in the south, and 79 in the east groups of registration districts. The weekly returns of the Metropolitan Asylums Board for the two years show that the number of completed cases of small-pox in their hospitals was 7242 in 1877 , and 4762 in 1878 . Thus, the ratio of mortality to cases in 1878 (17.6 per cent) was higher than that in $1877(17 \cdot 2)$. It is worthy of note that in the Highgate Small-pox Hospital the proportional mortality among the 422 completed cases recorded last year did not exceed $14 \cdot 2$ per cent. (British Medical fournal, 1879.) 